\title{
Mitotic-Chromosome-Based Physical Mapping of the Culex quinquefasciatus Genome
}

\author{
Anastasia N. Naumenko ${ }^{1}$, Vladimir A. Timoshevskiy ${ }^{1}$, Nicholas A. Kinney ${ }^{2}$, Alina \\ A. Kokhanenko ${ }^{3}$, Becky S. deBruyn ${ }^{4}$, Diane D. Lovin ${ }^{4}$, Vladimir N. Stegniy ${ }^{3}$, David \\ W. Severson ${ }^{4}$, Igor V. Sharakhov ${ }^{1}$, Maria V. Sharakhova ${ }^{1,3 *}$ \\ 1 Department of Entomology and Fralin Life Science Institute, Virginia Tech, Blacksburg, Virginia, United \\ States of America, 2 Department of Genomics, Bioinformatics, and Computational Biology, Virginia Tech, \\ Blacksburg, Virginia, United States of America, 3 Institute of Biology and Biophysics, Tomsk State \\ University, Tomsk, Russia, 4 Department of Biological Sciences and Eck Institute for Global Health, \\ University of Notre Dame, Notre Dame, Indiana, United States of America \\ *msharakh@vt.edu
}

\section{G openaccess}

Citation: Naumenko AN, Timoshevskiy VA, Kinney NA, Kokhanenko AA, deBruyn BS, Lovin DD, et al. (2015) Mitotic-Chromosome-Based Physical Mapping of the Culex quinquefasciatus Genome. PLoS ONE 10(3): e0115737. doi:10.1371/journal.pone.0115737

Academic Editor: Read Pukkila-Worley, University of Massachusetts Medical School, UNITED STATES

Received: November 14, 2014

Accepted: November 21, 2014

Published: March 13, 2015

Copyright: $\odot 2015$ Naumenko et al. This is an open access article distributed under the terms of the Creative Commons Attribution License, which permits unrestricted use, distribution, and reproduction in any medium, provided the original author and source are credited.

Data Availability Statement: BAC-end sequencing data are available using accession number LIBGSS_039177 within the Notre Dame Johannesburg BAC library at http://www.ncbi.nlm.nih. gov/. This information is also available within Table 1.

Funding: The research was funded by grant R21Al101345 to MVS from the National Institute of Allergy and Infectious Diseases, National Institute of Health www.niaid.nih.gov. The funders had no role in study design, data collection and analysis, decision to publish, or preparation of the manuscript.

\section{Abstract}

The genome assembly of southern house mosquito $C x$. quinquefasciatus is represented by a high number of supercontigs with no order or orientation on the chromosomes. Although cytogenetic maps for the polytene chromosomes of this mosquito have been developed, their utilization for the genome mapping remains difficult because of the low number of highquality spreads in chromosome preparations. Therefore, a simple and robust mitotic-chromosome-based approach for the genome mapping of $C x$. quinquefasciatus still needs to be developed. In this study, we performed physical mapping of 37 genomic supercontigs using fluorescent in situ hybridization on mitotic chromosomes from imaginal discs of 4th instar larvae. The genetic linkage map nomenclature was adopted for the chromosome numbering based on the direct positioning of 58 markers that were previously genetically mapped. The smallest, largest, and intermediate chromosomes were numbered as 1, 2, and 3, respectively. For idiogram development, we analyzed and described in detail the morphology and proportions of the mitotic chromosomes. Chromosomes were subdivided into 19 divisions and 72 bands of four different intensities. These idiograms were used for mapping the genomic supercontigs/genetic markers. We also determined the presence of length polymorphism in the $q$ arm of sex-determining chromosome 1 in $C x$. quinquefasciatus related to the size of ribosomal locus. Our physical mapping and previous genetic linkage mapping resulted in the chromosomal assignment of $13 \%$ of the total genome assembly to the chromosome bands. We provided the first detailed description, nomenclature, and idiograms for the mitotic chromosomes of $C x$. quinquefasciatus. Further application of the approach developed in this study will help to improve the quality of the southern house mosquito genome. 
Competing Interests: The authors have declared that no competing interests exist.

\section{Introduction}

Mosquito-borne infectious diseases pose unacceptable risks to public health and welfare [1]. Among mosquitoes, species of the genus Culex are the most taxonomically diverse and geographically widespread $[2,3]$. Mosquitoes within the $C x$. pipiens complex are major vectors for lymphatic filariasis caused by nematode Wuchereria bancrofti in tropical and subtropical regions of Asia, Africa, Central and South America, and Pacific Islands. Cx. quinquefasciatus is also a primary vector for arboviral infections, such as West Nile virus, St. Louis encephalitis, Sindbis, and Rift Valley fever viruses. Members of the Cx. pipiens complex have great variation in their host range, feeding behavior, and female diapause. Sequencing of the genomes for three major mosquito taxa, Anopheles gambiae [4], Aedes aegypti [5], and Cx. quinquefasciatus [6], provides important insights into genetic diversity of mosquitoes and evolution of the mosquito-pathogen interactions [7]. However, compared to other mosquitoes, Cx. quinquefasciatus has the most fragmented genome. A total of $579 \mathrm{Mb}$ (mega base pairs) is currently assembled into 3,171 supercontigs with the N50 size being $\sim 476 \mathrm{~Kb}$ (kilo base pairs). The N50 supercontig sizes are $12.3 \mathrm{Mb}$ in the An. gambiae (PEST) genome and $1.5 \mathrm{Mb}$ in the Ae. aegypti genome. A lack of a high-quality chromosome-based genome assembly for $C x$. quinquefasciatus remains a significant impediment to further progress in $C x$. quinquefasciatus biology and comparative genomics of mosquitoes. Fragmented unmapped genome assemblies create substantial problems for genome analysis. For example, unidentified gaps cause incorrect or incomplete annotation of genomic sequences; unmapped sequences lead to confusion between paralogous genes and genes from different haplotypes, and the lack of chromosome assignment and orientation of the sequencing contigs does not allow for studying chromosome organization and evolution [8]. Therefore, utility of the genome assembly for investigations on basic biology requires anchoring of the genomic supercontigs onto Cx. quinquefasciatus chromosomes.

Among other approaches, genetic linkage mapping has been so far the most effective method for the genome mapping of $C x$. quinquefasciatus. Among closely related $C x$. pipiens species, several morphological mutants have been described by Leonore Dennhofer [9]. Crosses involving different mutants permitted assignment of genes related to these mutations to three linkage groups. Use of deoxyribonucleic acid (DNA) markers as a new approach allowed the construction of a genetic map which originally consisted of 21 complementary DNA (cDNA) markers and covered 7.1, 80.4, and 78.3 cM on chromosomes 1, 2, and 3, respectively [10]. The sex determination locus was genetically mapped to the smallest linkage group 1 in Cx. pipiens. In addition, multiple quantitative trait loci (QTL), related to differences in reproductive diapause between species in the $C x$.pipiens complex, were also genetically mapped [11]. The most recent genetic linkage map developed for Cx. quinquefasciatus includes 63 genetic loci [12]. This map covered 29.5, 88.8, and $65.6 \mathrm{cM}$ on linkage groups 1, 2, and 3 and allowed integration of $10.4 \%$ of the genome with the genetic linkage map. Currently, this is the most representative map of the Cx. quinquefasciatus genome. However, this map has never been integrated with cytogenetic maps developed for this mosquito.

Physical mapping in Cx. quinquefasciatus is challenging because of the poor quality of the polytene chromosomes. Several attempts to create a cytogenetic photomap using $C x$. quinquefasciatus polytene chromosomes have been made. The Malpighian tubule chromosome map for Cx. pipiens [13] and Cx. quinquefasciatus [14] and, more recently, the salivary gland chromosome map for Cx. quinquefasciatus [15], were developed. However, correspondence of arms and regions among these maps and the original drawn map published by L. Dennhofer [16] is uncertain. Almost no similarities between landmarks of different chromosome maps were found [15]. These problems occurred because of low levels of polyteny, high frequency of ectopic contacts or associations of nonhomologous chromosome regions, and poor spreading of 
Cx. quinquefasciatus polytene chromosomes in preparation. As a result, only two genes for esterase- and odorant-binding proteins were mapped to the polytene chromosomes of $C x$. quinquefasciatus $[15,17]$.

In contrast to polytene chromosomes, mitotic chromosomes do not form ectopic contacts and can be easily utilized for mapping DNA probes to the chromosome bands. A simple and robust technique for obtaining high-quality mitotic chromosomes from imaginal discs of $4^{\text {th }}$ instar larvae was recently developed for the yellow fever mosquito Ae. aegypti [18]. This work resulted in 13\%, and more recently, in $45 \%$ of the genome placement to the chromosomes for this mosquito [19,20]. Mitotic chromosomes of Cx.pipiens, the closest relative of $C x$. quinquefasciatus, have been briefly described in 1963 as three pairs of metacentric chromosomes and numbered in order of increasing size as chromosomes I, II, and III [21]. In some cases, chromosome I was identified as a submetacentric chromosome, meaning that the relative length of the shorter arm $\mathrm{p}$ was less than $35 \%$ of the total chromosome length. It was also determined that $C x$. pipiens chromosomes are smaller than those in Ae. aegypti. Chromosome measurements also demonstrated that compared with Ae. aegypti chromosomes $C x$. pipiens chromosome I was disproportionally smaller than chromosomes II and III. Unlike in anophelines that have heteromorphic X and Y sex chromosomes [22], sex-determining chromosomes in $C x$. quinquefasciatus were considered as homomorphic. Only two genes, $18 \mathrm{~S}$ and $28 \mathrm{~S}$ ribosomal DNA (rDNA), have been physically mapped to the smallest mitotic chromosome of $C x$. pipiens [23]. Chromosome maps suitable for the physical mapping have not been developed for the mitotic chromosomes of $C x$. quinquefasciatus.

In this study, mitotic chromosomes of $C x$. quinquefasciatus were described in details and directly linked to the previously established genetic linkage groups by hybridization of 26 Bacterial Artificial Chromosome (BAC) probes associated with 58 genetic markers [24] to the chromosomes. As a result, chromosomes were renumbered according to the existing genetic linkage groups $[10,12]$. We also developed idiograms or schematic representation of chromosome banding patterns for $C x$. quinquefasciatus. In addition, we mapped an $18 \mathrm{~S}$ rDNA probe and 9 large genomic supercontigs to the chromosomes. Thus, our study has demonstrated that a mitotic chromosome band-based technique can be utilized for further development a highresolution physical genome map for the $C x$. quinquefasciatus.

\section{Materials and Methods}

\section{Mosquito strain and slide preparation}

The laboratory strain Johannesburg (JHB), used in this study was obtained from BEI Resources [25]. This colony was originated from the field population of $C x$. quinquefasciatus near Johannesburg, South Africa [15]. The same strain was previously used for the genome sequencing project [6]. Adult mosquitoes were kept at $26^{\circ} \mathrm{C}$ and fed on artificial membrane blood feeders $4-5$ days after emerging. Approximately 4 days after feeding, the eggs were collected and hatched at $26^{\circ} \mathrm{C}$. After 4 days, $2^{\text {nd }}$ instar larvae were transferred to $16^{\circ} \mathrm{C}$ to obtain a high number of mitotic divisions in imaginal discs [18]. At 7-8 days, $4^{\text {th }}$ instar larvae were used for slide preparation. Our study utilized mitotic chromosomes from imaginal discs of $4^{\text {th }}$ instar larvae which develop into legs and wings at the adult stage. These imaginal discs are located immediately under the cuticle and can be easily dissected from the larvae. The morphology of the imaginal discs and details of their dissection from the larvae were previously described for three species of mosquitoes including $C x$. quinquefasciatus [26]. Chromosome preparations were made using a routine technique based on hypotonic treatment and subsequent application of Carnoy's solution (3 parts of ethanol: 1 part of acetic acid) and 50\% propionic acid [26]. 
The percentage of chromosome preparations suitable for further analysis, which contained more than 50 chromosome spreads, was $\sim 85 \%$.

\section{DNA probe preparation and fluorescent in situ hybridization (FISH)}

Notre Dame Johannesburg (NDJ) BAC library [24] was used as a probe DNA source for FISH. BAC clone DNA isolation and sequencing were performed at the Clemson University Genomics Institute. BAC clone correspondence to the certain genomic supercontigs or genetic markers was determined by BAC library screening [26] or by BAC-end sequence comparison using Basic Local Alignment Search Tool (BLAST) against the genome assembly of $C x$. quinquefasciatus available at Vectorbase [27]. Three polymerase chain reaction (PCR) fragments with sizes $\sim 1 \mathrm{~Kb}$ from genomic supercontig 3.32 were amplified using primers: AAAACCC ATCTCCCTCGTAG forward, GCTTCTCCAAAACCTTCCTC reverse; TCAAACGACCAC AACTTTGA forward, TGGCCTTGTTCTTCTTCTTG reverse; and ATGAAGTTACGGTC GTCAGC forward, AGTGCATGATGACTCCCATT reverse. Probes were labeled by nick translation with Cy3- or Cy5-deoxyuridine 5-triphosphate (dUTPs) (GE Healthcare UK Ltd., Buckinghamshire, UK) as described before [26]. An 18S rDNA probe was amplified using forward primer CCTATATGGTGGCGCTTGAT and reverse primer AACTAAGAACGGCC ATGCAC. It was labeled by Cy3- or Cy5-dUTPs in a PCR reaction using PCR IMMOMIX (Bioline USA, Taunton, MA) with standard parameters. Nonspecific hybridization of BAC DNA probes to the chromosomes was prevented by pre-hybridization of the probe with unlabeled repetitive DNA fractions of genomic DNA [26]. Genomic DNA was extracted from adult mosquitoes using Qiagen Blood \& Cell Culture DNA Maxi Kit (Qiagen Science, Germantown, MD, USA). Approximately $500 \mathrm{mg}$ of adult mosquitoes were taken for extraction. Final outcome of repetitive DNA fractions accounts for $\sim 20 \%$ of genomic DNA. Approximately $250-$ $350 \mathrm{ng}$ of DNA probe were pre-hybridized with $4 \mathrm{mg}$ of repetitive DNA. FISH of DNA probes was performed using a standard protocol [26]. Slides were analyzed using Zeiss LSM 510 Laser Scanning Microscope (Carl Zeiss Microimaging, Inc., Thornwood, NY, USA) at 600X magnification. For each probe, from 5-10 chromosome spreads were tested.

\section{Image processing and measurements}

For idiogram development, the best images of the chromosomes from imaginal discs stained with Oxasole Yellow (YOYO-1) iodide (Invitrogen Corporation, Carlsbad, CA, USA) were selected. The original images were converted into gray-scale images and contrasted as described previously [28]. These chromosome images were straightened and aligned for comparison using ImageJ program $[18,29]$. In total, 150 chromosomes at early metaphase were analyzed, and 25-30 images of each chromosome with reproducible banding patterns were used for idiogram development. To calculate exact proportions of chromosomes, we utilized standard curve measurements in Zen2009LightEdition software [30]. We utilized early metaphase and midmetaphase chromosomes to precisely assign signals to the particular chromosome band.

\section{Results}

\section{Culex quinquefasciatus chromosome nomenclature}

According to an original chromosome nomenclature, three pairs of metacentric chromosomes of $C x$. pipiens, the closest relative of $C x$. quinquefasciatus, were numbered as I, II, and III in order of increasing size (Rai, 1963). In this study, we established correspondence between mitotic chromosomes and genetic linkage groups by direct placement of 26 genomic supercontigs associated with 58 genetic markers to the chromosomes (Table 1). These markers were 
Table 1. List of genomic supercontigs, BAC clones, and genetic markers mapped to the chromosome of Cx. quinquefasciatus.

\begin{tabular}{|c|c|c|c|c|c|c|}
\hline SC & SC size & BAC well/plate & $\mathrm{AC \#}(\mathrm{T} 7)$ & AC\# (M13) & Genetic marker (AC\#) & Location \\
\hline 3.845 & 193967 & NDJ.020P4 & GF110931 & $\mathrm{N} / \mathrm{A}$ & $\mathrm{N} / \mathrm{A}$ & $1 \mathrm{p} 12$ \\
\hline 3.14 & 1835525 & NDJ.001J24 & KG777556 & $\mathrm{N} / \mathrm{A}$ & $\mathrm{N} / \mathrm{A}$ & $1 \mathrm{p} 21$ \\
\hline 3.36 & 1496554 & NDJ.003F11 & KG961588 & KG961589 & C36GTT1 & $1 \mathrm{p} 31$ \\
\hline 3.12 & 1895535 & NDJ.003N20 & KG961610 & KG961611 & C12CCT1, C12GTT1, C12GTC, Cxpq51 & $1 \mathrm{p} 33$ \\
\hline 3.56 & 1226699 & NDJ.001010 & KG777559 & KG777560 & LF284 (BM005502), C56GCA1, C56TGT1 & $1 q 12$ \\
\hline 3.134 & 821072 & NDJ.001D17 & KG777561 & KG777562 & $\mathrm{C} 134 \mathrm{AC} 1 \mathrm{~b}$ & $1 q 12$ \\
\hline 3.1464 & 65637 & $\mathrm{~N} / \mathrm{A}$ & $\mathrm{N} / \mathrm{A}$ & $\mathrm{N} / \mathrm{A}$ & $18 \mathrm{~S}$ rDNA & $1 q 13$ \\
\hline 3.49 & 1394590 & NDJ.002A07 & KG961590 & KG961591 & $\mathrm{N} / \mathrm{A}$ & $1 q 21$ \\
\hline 3.127 & 851360 & NDJ.048G24 & $\mathrm{N} / \mathrm{A}$ & $\mathrm{N} / \mathrm{A}$ & CX60 (FD664718) & $1 q 31$ \\
\hline 3.129 & 873558 & NDJ.064F11 & $\mathrm{N} / \mathrm{A}$ & $\mathrm{N} / \mathrm{A}$ & CX107 (FD664723), C129CT1 & $2 p 25$ \\
\hline 3.280 & 510693 & NDJ.001J13 & KG961592 & KG961593 & C28GT1 & $2 p 31$ \\
\hline 3.66 & 1106043 & NDJ.002N10 & KG961586 & KG961587 & C66CGT1, C66GT1, C66TGT1 & $2 p 32$ \\
\hline 3.15 & 1741670 & NDJ.002C09 & KG777563 & KG777564 & N/A & $2 p 34$ \\
\hline 3.177 & 728683 & NDJ.001N13 & KG961594 & KG961595 & CX114 (FD664728), C177CGT1 & $2 p 34$ \\
\hline 3.186 & 747982 & NDJ.001K16 & KG961596 & KG961597 & C186TGT1 & 2p35 \\
\hline 3.5 & 2487969 & NDJ.002C07 & KG777565 & KG777566 & CX44 (FD664710), C5CGT1, C5GTG1 & $2 q 11$ \\
\hline 3.11 & 2034973 & NDJ.001N24 & KG777567 & KG777568 & N/A & $2 q 12$ \\
\hline 3.9 & 2056888 & NDJ.001E14 & KG777571 & KG777572 & $\mathrm{N} / \mathrm{A}$ & $2 q 13$ \\
\hline 3.17 & 1689851 & NDJ.001E04 & KG777569 & KG777570 & $\mathrm{N} / \mathrm{A}$ & $2 q 17$ \\
\hline 3.95 & 956384 & NDJ.003F24 & KG961598 & KG961599 & LF203 (BM005503), C95CAG1, C95GCA1 & $2 q 22$ \\
\hline 3.4 & 2511003 & NDJ.002L02 & KG961600 & KG961601 & C4TTG1 & $2 q 25$ \\
\hline 3.18 & 1726395 & NDJ.001D20 & KG777573 & KG777574 & N/A & $2 q 32$ \\
\hline 3.10 & 2129711 & NDJ.001J08 & KG777575 & KG777576 & $\mathrm{N} / \mathrm{A}$ & $2 q 32$ \\
\hline 3.68 & 1113402 & N/A & N/A & N/A & LF335 (BM005505), C68TCG1 & $2 q 42$ \\
\hline 3.13 & 1876709 & NDJ.003H21 & KG961602 & KG961603 & C13TC1 & $2 q 43$ \\
\hline 3.32 & 1521851 & NDJ.033G10 & N/A & N/A & LF334 (BM005506), C32AC1, C32AG1, С32TC1b, C32TGC1 & $2 q 43$ \\
\hline 3.65 & 1116611 & NDJ.005K08 & $\mathrm{N} / \mathrm{A}$ & KG961581 & C65AC1, C65CAG1 & $3 p 11$ \\
\hline 3.446 & 375653 & NDJ.009F06 & $\mathrm{N} / \mathrm{A}$ & N/A & CX11 (FD664697), C446TC1 & $3 p 32$ \\
\hline 3.208 & 649753 & NDJ.003E21 & N/A & $\mathrm{N} / \mathrm{A}$ & C208GCA1, CxqTri4 & 3p32 \\
\hline 3.1 & 3873040 & NDJ.001G13 & KG777581 & KG777582 & C1CAG1 & $3 q 12$ \\
\hline 3.67 & 1097170 & NDJ.002F04 & N/A & N/A & LF108 (T58322), C67CT1 & $3 q 12$ \\
\hline 3.73 & 1095011 & NDJ.004O20 & KG961605 & KG961605 & LF272 (BM005484), C73CA1, C73TCG1 & $3 q 14$ \\
\hline 3.139 & 823831 & NDJ.013B09 & $\mathrm{N} / \mathrm{A}$ & N/A & CX53 (FD664714), C139CT1, C139GA1 & $3 q 21$ \\
\hline 3.205 & 667856 & NDJ.005A07 & KG961612 & KG961613 & CX17 (FD664699), C205GAC1, C205GTC1 & $3 q 21$ \\
\hline 3.99 & 949261 & NDJ.024B18 & $\mathrm{N} / \mathrm{A}$ & $\mathrm{N} / \mathrm{A}$ & CX112 (FD664727), C99GTC1 & $3 q 24$ \\
\hline 3.2 & 2744360 & NDJ.002F15 & KG961606 & KG961607 & C2ACG1 & $3 q 32$ \\
\hline 3.3 & 2758190 & NDJ.001M21 & KG777583 & KG777584 & LF115 (R67978), С3GAC1, С3TGC1 & $3 q 33$ \\
\hline
\end{tabular}

N/A-not applicable

doi:10.1371/journal.pone.0115737.t001

previously mapped to smallest, largest, and intermediate linkage groups 1, 2, and 3 of $C x$. pipiens, respectively [10]. Seven BAC clones for this mapping were identified by screening the NDJ BAC library [24] using PCR-amplified genetic markers [31]. Another 19 BAC clones were identified as belonging to the genomic supercontigs with known genetic markers by BAC-end sequencing followed by BLAST-based alignment to the genomic sequences of $C x$. quinquefasciatus [27]. BAC clones corresponding to linkage group 1 carrying markers CX60, LF284, and 
Table 2. The measurements of $\mathrm{Cx}$. quinquefasciatus mitotic chromosomes from imaginal discs in comparison to Ae. aegypti.

\begin{tabular}{lll}
\hline Mosquito species & Cx. quinquefasciatus & Ae. aegypti \\
\hline Chromosome 1 Average length, $\mu \mathrm{m}$ & 4.04 & 7.1 \\
Relative length, \% & $25.30 \%$ & $28.6 \%$ \\
Centromeric index, \% & $43.1 \%$ or $48.1 \%$ & $46.9 \%$ \\
Chromosome 2 Average length, $\mu \mathrm{m}$ & 6.37 & 9.5 \\
Relative length, \% & $39.80 \%$ & $37.9 \%$ \\
Centromeric index, \% & $47.4 \%$ & $48.6 \%$ \\
Chromosome 3 Average length, $\mu \mathrm{m}$ & 5.59 & 8.4 \\
Relative length, \% & $34.9 \%$ & $33.5 \%$ \\
Centromeric index, \% & $46.9 \%$ & $47.4 \%$ \\
\hline
\end{tabular}

doi:10.1371/journal.pone.0115737.t002

8 microsatellite markers were mapped to the smallest chromosome. Markers consisting of 6 complementary DNA (cDNA) and 16 microsatellites from linkage group 2 were mapped to the largest chromosome. Seven cDNA and 17 microsatellite markers from linkage group 3 were mapped to the intermediate-in-size chromosome. Supercontig 3.32 containing genetic marker LF335 was mapped as 3 PCR-amplified products with sizes $\sim 1 \mathrm{~Kb}$. The order of most markers on chromosomes exactly followed their positions in the genetic linkage map. We found only two discrepancies in the order of markers CX44 and LF203 on chromosome 2 and also a BAC clone with genetic marker LF108 was mapped on a different chromosome. Thus, we propose renumbering the mitotic chromosomes for $C x$. quinquefasciatus in correspondence to the genetic linkage groups as follows: 1 -smallest, 2 -largest, and 3-intermediate chromosomes.

We also determined the average chromosome lengths at mid-metaphase as $4.04 \mu \mathrm{m}$ for chromosome 1, $6.37 \mu \mathrm{m}$ for chromosome 2, and $5.59 \mu \mathrm{m}$ for chromosome 3 (Table 2). Relative lengths of chromosomes were $25.3 \%, 39.8 \%$, and $34.9 \%$ for each chromosome, respectively. Centromeric indexes (the relative length of the p-arm) were $47.4 \%$ and $46.9 \%$ for chromosomes 2 and 3. Thus our data confirmed that these two chromosomes are metacentric [21]. Measurements for the centromere position in chromosome 1 varied depending on size of the ribosomal locus determined by FISH of $18 \mathrm{~S}$ rDNA probe on the chromosome between $43.1 \%$ or $48.1 \%$, respectively (Fig. 1). Thus, both variants of chromosome 1 must be also considered as metacentric according to the modern chromosome nomenclature [32].
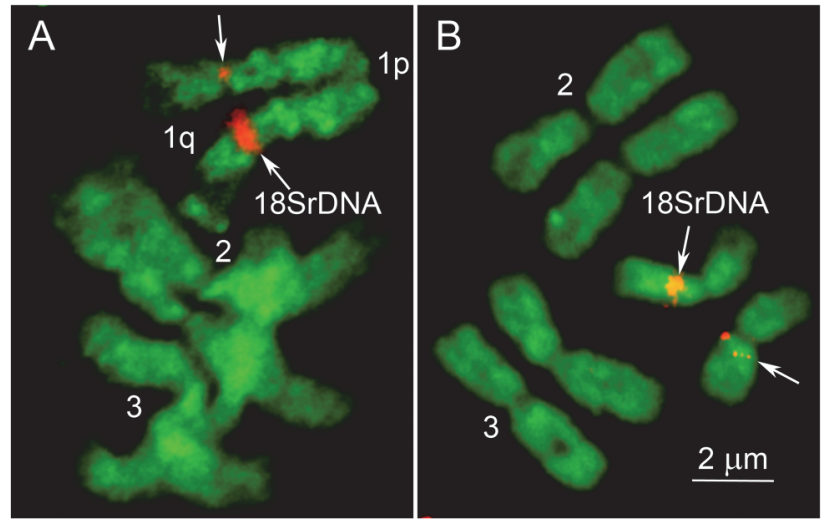

Fig 1. Two variants of sex-determining chromosome 1 at early- (A) and mid-metaphase (B). Position of 18S rDNA probe on chromosome 1 is indicated by arrow.

doi:10.1371/journal.pone.0115737.g001 

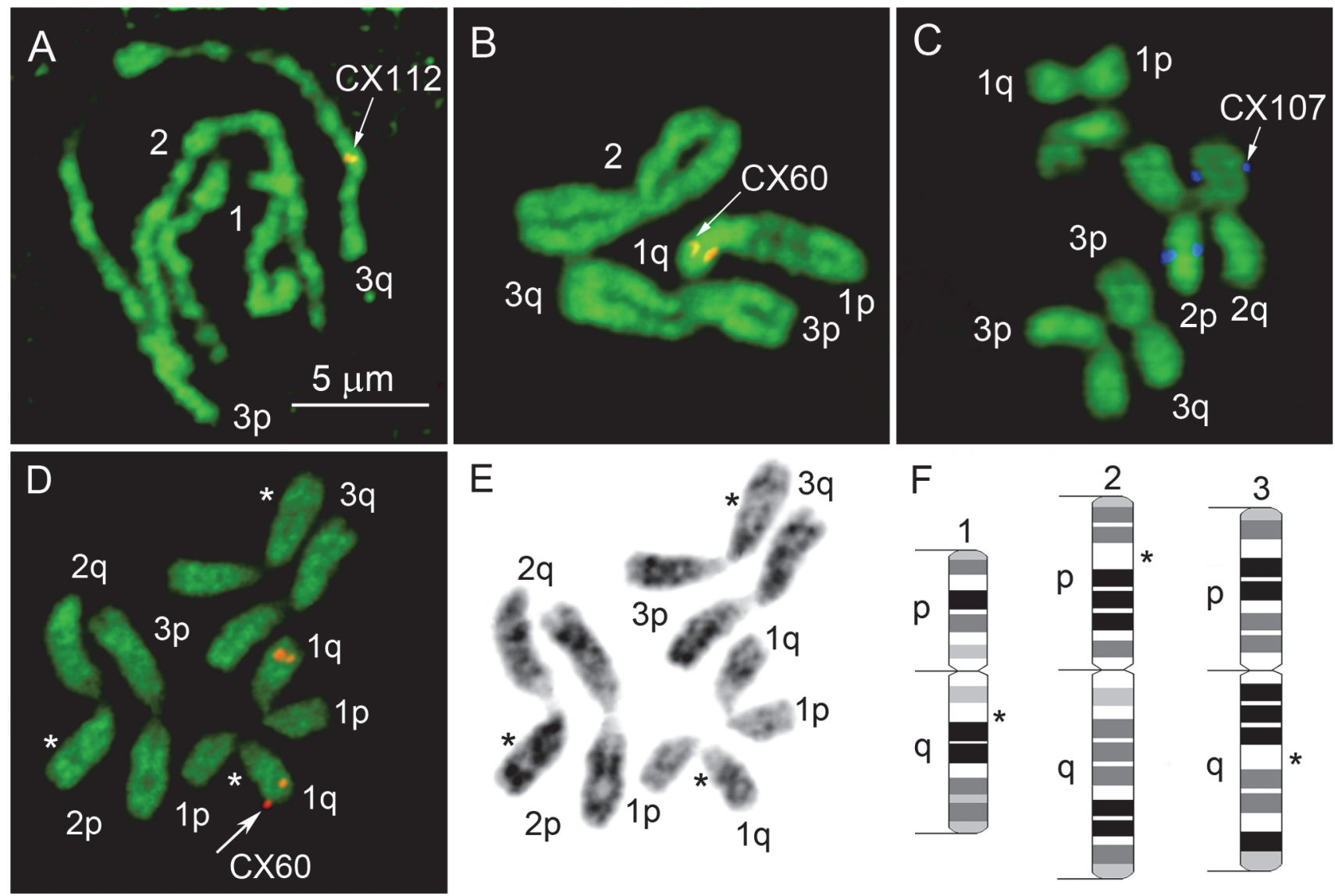

Fig 2. Stages of mitosis (A-C) and chromosome idiogram development (D-F) in Cx. quinquefasciatus. Early metaphase chromosomes (D) were chosen from prophase (A), prometaphase $(B)$, and late-metaphase $(C)$ chromosomes for the ideogram development. Chromosome images stained with YOYO-1 iodide were converted into gray images (E). Chromosomes on idiograms were subdivided into 72 bands with 4 different intensities (F). Arrows show chromosome positions of the genetic markers CX60 (B, D), CX 112 (A), and CX107 (C). Chromosome landmarks are indicated by asterisks.

doi:10.1371/journal.pone.0115737.g002

\section{Idiograms of mitotic chromosomes for Culex quinquefasciatus}

In addition to chromosome nomenclature, our study developed idiograms or drawn schematic representations of the banding pattern for mitotic chromosomes of $C x$. quinquefasciatus at early-metaphase. From a whole range of the different stages of mitosis (prophase, prometaphase, metaphase, and anaphase), metaphase chromosomes have the most clear and reproducible banding patterns (Fig. 2). Similarly to Ae. aegypti, in Cx. quinquefasciatus homologous chromosomes are paired at prophase and prometaphase (Fig. 2A, B). At these two stages, the visible chromosome number equals three. Chromosomes start segregating from each other at prometaphase and become completely segregated at metaphase (Fig. 2C). Visible chromosome number at metaphase equals six.

For the idiogram development, we used images of the chromosomes at early metaphase stained with YOYO-1 iodide (Fig. 2D). This dye strongly stains euchromatin and therefore provides more detailed banding patterns than DAPI (4',6-diamidino-2-phenylindole), which preferentially stains AT-rich heterochromatic regions $[18,26]$. The original chromosome pictures were converted into gray-scale images. Chromosome images were then straightened and aligned for comparison. After that, unique and reproducible patterns for each chromosome were identified. Following human chromosome nomenclature [33], we determined four color 


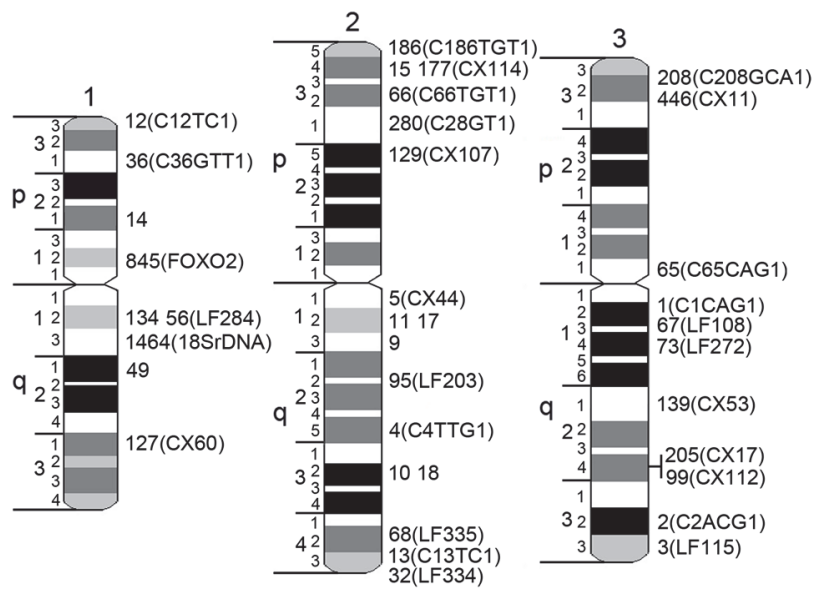

Fig 3. A landmark-guided two-step physical mapping approach on Cx. quinquefasciatus chromosomes. FISH of two BAC clones of interest was performed in the presence of 2 additional BAC clones, and 18S rDNA used as landmarks for the chromosome arm identification (A-C). Positions of molecular landmarks and 2 BAC clones of interest are indicated by arrows. Mitotic chromosomes at metaphase were used for the rapid assignment of the genomic supercontigs to the chromosome bands (D). Longer prophase $(E)$ or polytene chromosomes $(F)$ were further utilized for ordering the genomic supercontigs within the band.

doi:10.1371/journal.pone.0115737.g003

intensities of the chromosome bands: intense (black), medium intensity (dark gray), low intensity (light gray), and negative (white). Chromosomes were subdivided into 20, 28, and 24 bands for chromosomes 1,2, and 3, respectively (Fig. 2E). The total number of bands for all chromosomes of Cx.quinquefasciatus equals 72. Each chromosome of $C x$. quinquefasciatus has unique features or landmarks for the arm recognition: large negative band containing ribosomal locus in the $\mathrm{q}$ arm region of chromosome 1 , negative band separating intense and mediumintense bands in the $\mathrm{p}$ arm of chromosome 2 , and large negative band in the middle of arm $\mathrm{q}$ on chromosome 3 (Fig. 2).

\section{Physical mapping on mitotic chromosomes of Culex quinquefasciatus}

In addition to BAC clones associated with genetic markers, $9 \mathrm{BAC}$ clones from the largest genomic supercontigs and $18 \mathrm{~S}$ ribosomal DNA (Table 2) were also mapped to the bands on idiograms by FISH (Fig. 3). An 18 S ribosomal DNA probe hybridized above the 2 dark bands on the $\mathrm{q}$ arm of chromosome 1 in region 1q13 on the idiogram. In total, a majority of the DNA probes (17) hybridized to the largest chromosome 2, 9 BAC clones were found in intermediatesized chromosome 3 , and 11 DNA probes hybridized to the smallest sex-determining chromosome 1. To simplify physical mapping, we optimized a landmark-guided approach developed for Ae. aegypti $[20,34]$ for Cx. quinquefasciatus chromosomes (Fig. 4). We hybridized two BAC clones of interest in the presence of 3 landmark probes: $18 \mathrm{~S} \mathrm{rDNA}$ for $1 \mathrm{q}$ arm, telomere BAC clone with genetic marker LF334 on 2q arm, and a BAC clone with genetic marker CX112 close to telomere for $3 \mathrm{q}$ arm. Two BAC clones on $3 \mathrm{q}$ arm carrying genetic markers CX17 and CX112 were ordered within the band on 3q arm using a two-step mapping approach [19]. In addition to FISH on metaphase mitotic chromosomes (Fig. 4D), the FISH results on prophase and polytene chromosomes were also analyzed. This additional step permitted the ordering of these genetic markers within chromosome band (Fig. 4E, F). 

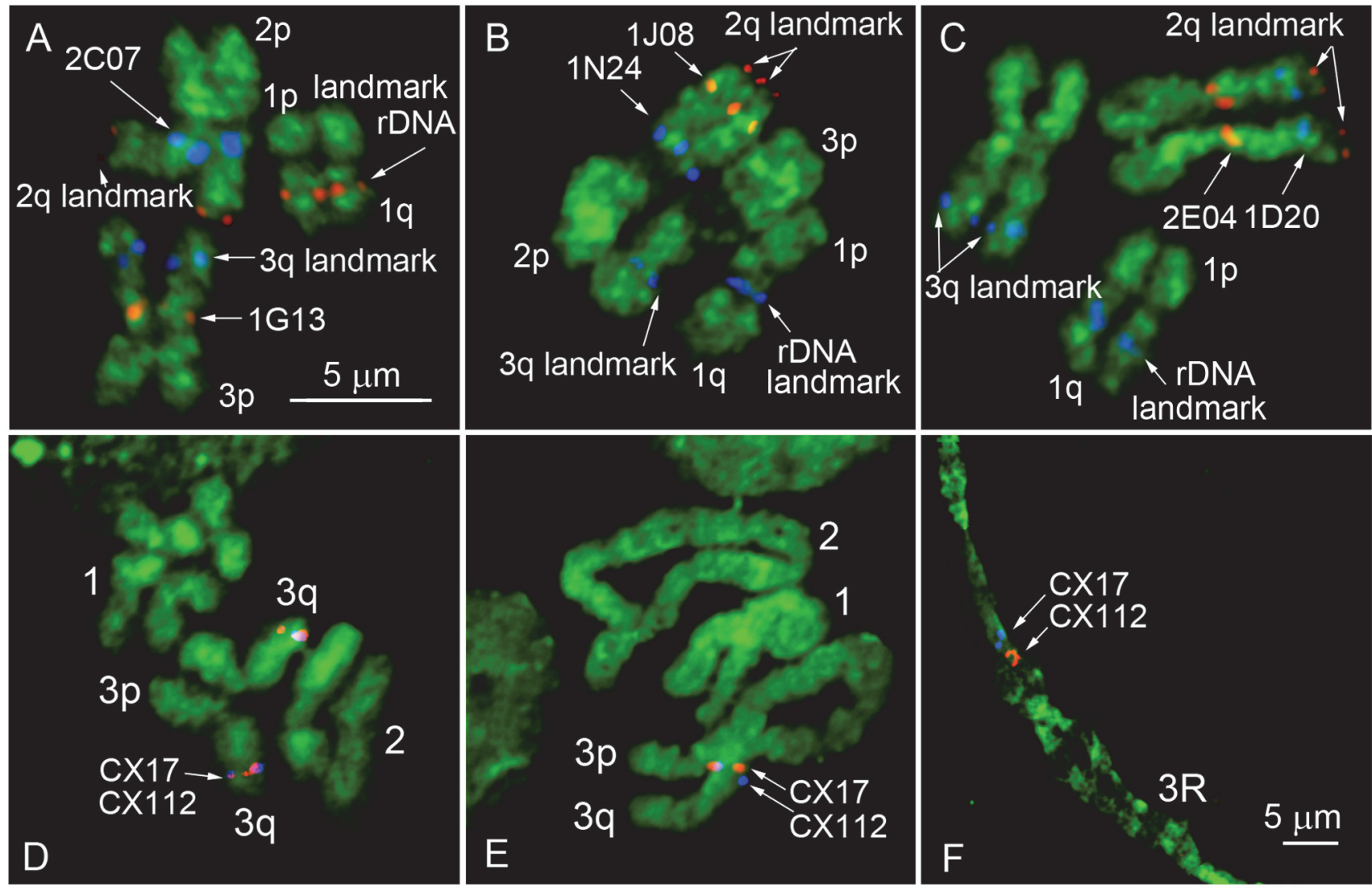

Fig 4. Chromosome idiograms with positions of supercontigs and genetic markers. Chromosomes 1, 2, and 3 are indicated by numbers. Short and long chromosome arms are indicated by letters $p$ and q, respectively. Chromosomes are subdivided into 19 divisions and 72 bands. Genomic supercontigs are indicated by the last 1 to 4 digits of their accession numbers. Genetic markers are shown in brackets.

doi:10.1371/journal.pone.0115737.g004

\section{Discussion}

Knowledge about cytogentics of mosquitoes is important to better understand their genome organization and function. However, mitotic chromosomes for the major vector of lymphatic filariasis $C x$. quinquefasciatus were only briefly mentioned, as three pairs of metacentric chromosomes, more than 50 years ago in a review on mosquito mitotic chromosomes [21]. For other mosquitoes such as An. gambiae and Ae. aegypti, chromosome work was performed back in the 1970s and 1980s, but it was not done for the Cx. quinquefasciatus. Here, for the first time, we described the details of morphology, length, and proportions for the mitotic chromosomes of $C x$. quinquefasciatus. Our measurements demonstrated that a total chromosome length at mid-metaphase in Cx. quinquefasciatus is 1.5 times longer than in An. gambiae and 1.5 times shorter than in Ae. aegypti (Table 2) [26]. It reflects the difference in genome sizes of $264 \mathrm{Mb}, 579 \mathrm{Mb}$, and $1376 \mathrm{Mb}$ in An. gambiae, Cx. quinquefasciatus, and Ae. aegypti, respectively. Our measurements also indicated the presence of two variants of sex-determining chromosome 1 in $C x$. quinquefasciatus that differ from each other by the size of ribosomal locus and the centromere position on this chromosome. This polymorphism can be potentially related to the sex determination in this mosquito.

Our study also, for the first time, integrated the cytogenetic map with the genetic linkage map $[10,12]$ and created a new chromosome nomenclature for $C x$. quinquefasciatus. Originally numbered as I, II, and III in order of increasing size [21], chromosomes were renumbered as 
1-smallest, 2-largest, and 3-intermediate chromosomes in correspondence to the genetic linkage map of $C x$. quinquefasciatus. The correspondence between genetic linkage groups and chromosomes was determined by direct positioning of 58 genetic markers on the chromosomes. The order of the markers on the chromosomes were basically the same as on the previously published genetic linkage map [12] with only two exceptions in order of the markers CX44 and LF203 on chromosome 2 and position of the genetic marker LF108 on a different chromosome. For Ae. aegypti, linkage groups were associated with chromosomes based on the analysis of X-ray-generated chromosome translocations in 1970 [35]. The smallest, largest, and intermediate chromosomes were also renumbered as 1,2, and 3, according to the genetic linkage groups. Recent physical mapping of 100 genetic markers of Ae. aegypti helped to clarify the chromosome positions of 12 QTL related to pathogen transmission: the filarioid nematode [36], the avian malaria parasite [37,38], and dengue virus [39,40]. These markers were combined into five major clusters of QTL on the chromosome map suggesting that transmission of various pathogens can be controlled by the same genomic loci [19]. However, QTL related to the pathogen transmission for Cx. quinqufasciatus have not been identified yet. Only multiple QTL to diapause were described [11]. Thus, further development of integrated genetic linkage and chromosome map requires both physical mapping and additional QTL identification.

In addition to chromosome nomenclature, our study developed detailed idiograms for the $C x$. quinquefasciatus chromosomes. The whole chromosome complement was subdivided into 19 regions and 72 bands with four different intensities. We demonstrated the utility of our idiograms for the physical genome mapping by placement of 37 genomic supercontigs to the chromosome locations. Chromosome idiograms for $C x$. quinquefasciatus are comparable to that previously developed for the yellow fever mosquito Ae. aegypti [18]. The total number of bands (72) is slightly lower in Cx. quinquefasciatus than in Ae. aegypti (94). Physical mapping approach based on idiograms allowed assignment of $45 \%$ of the Ae. aegypti genome to chromosome bands [20]. Additional physical mapping, based on the idiograms developed by this study for Cx. quinquefasciatus, needs to be conducted to increase the genome assignment to the chromosome position.

Using mitotic chromosomes for physical genome mapping raises a concern about the low resolution of this mapping approach compared to traditionally used polytene chromosomes [41-44]. Unlike the subfamily Anophelinae, which have well-developed polytene chromosomes, mosquitoes from the Culicinae subfamily lack high-quality polytene chromosome spreads $[14,45]$. Nevertheless, our study determined that in addition to mitotic chromosomes low-polytenized chromosomes from salivary glands can be used for the ordering of closely located supercontigs of $C x$. quinquefasciatus without assigning them to specific bands in polytene chromosomes (Fig. 2). This so called "two-step" mapping approach was successfully used for the ordering of 100 genomic supercontigs in Ae. aegypti [19]. This strategy significantly increased the final resolution of the physical map. The distance between two signals that can be distinguished from each other was estimated at $300 \mathrm{~Kb}$ for the polytene chromosomes of $\mathrm{Ae}$. aegypti. The resolution of polytene chromosomes in Cx. quinquefasciatus is higher due to their better polytenization and comparable to the $100-\mathrm{Kb}$ resolution of polytene chromosomes in An. gambiae [42].

Previous investigations of chromosome arm homology between Cx. quinquefasciatus, An. gambiae, and Ae. aegypti indicated whole-arm conservation between $C x$. quinquefasciatus and An. gambiae, and a whole-arm translocation between chromosomes 2 and 3 of $C x$. quinquefasciatus and Ae. aegypti [6]. This conclusion was based only on $9 \%, 31 \%$, and $88 \%$ genome placement to the chromosomes for Cx. quinquefasciatus, Ae. aegypti, and An. gambiae, respectively $[10,42,46]$. The dramatic gene order reshuffling between homologous chromosomes of Ae. aegypti and An. gambiae was recently demonstrated based on $45 \%$ and $88 \%$ genome placement 
to the chromosomes for these two mosquitoes, respectively [20]. Additional physical mapping may provide some new insights into chromosome evolution in Cx. quinquefasciatus. For example, FISH results for $18 \mathrm{~S}$ rDNA suggest an inverted position of the ribosomal locus in chromosome 1 of Cx. quinquefasciatus compared with Ae. aegypti [19]. This locus was mapped close to the centromere above the dark bands in Cx. quinquefasciatus (Fig. 1) but in the middle of the 1q arm below the dark band in Ae. aegypti. Our measurement data of $C x$. quinquefasciatus chromosomes support the previous observation that the proportions between sex-determining chromosome 1 and autosomes 2 and 3 differ in Cx. pipiens and Ae. aegypti (Table 2). It is clear that the relative length of chromosome 1 is shorter in Cx. quinquefasciatus than in Ae. aegypti. These results support the idea of partial degradation of the sex-determining chromosome 1 in Cx. quinquefasciatus compared to chromosome 1 in Ae. aegypti. Degradation of sex chromosomes was described in different lineages of Drosophila [47]. However, a more advanced chromosome-based genome map for $C x$. quinquefasciatus is required for clarifying the intimate details of chromosome evolution in mosquitoes.

\section{Conclusion}

Our study developed a mitotic-chromosome-based approach for physical mapping of the $C x$. quinquefasciatus genome. We provided the first detailed description and offered a new nomenclature for mitotic chromosomes of $C x$. quinquefasciatus. Based on the genetic linkage map, the smallest, largest, and intermediate chromosomes were numbered as 1,2, and 3, respectively. We demonstrated the efficiency of our physical mapping approach by placing 37 genomic supercontigs and 58 genetic markers onto chromosome idiograms. This effort, together with previously conducted linkage mapping [12], resulted in the chromosome assignment of $13 \%$ of the total genome assembly. Further application of the approach described here will improve the current highly fragmented genome assembly of $C x$. quinquefasciatus and will also stimulate research in vector biology and comparative genomics in mosquitoes.

\section{Acknowledgments}

Authors thank Sergei Demin for the image processing, Joanne Cunningham for the technical help, and Melissa Wade for editing the manuscript.

\section{Author Contributions}

Conceived and designed the experiments: MVS IVS. Performed the experiments: ANN VAT BSD DDL NAK AAK. Analyzed the data: ANN MVS. Contributed reagents/materials/analysis tools: DWS VNS. Wrote the paper: ANN MVS IVS DWS.

\section{References}

1. Tolle MA (2009) Mosquito-borne diseases. Curr Probl Pediatr Adolesc Health Care 39: 97-140. doi: 10.1016/j.cppeds.2009.01.001 PMID: 19327647

2. Reddy BP, Labbe P, Corbel V (2012) Culex genome is not just another genome for comparative genomics. Parasit Vectors 5: 63. doi: 10.1186/1756-3305-5-63 PMID: 22463777

3. Vinogradova EB (2000) Culex pipiens pipiens mosquitoes: taxonomy, distribution, ecology, physiology, genetics, applied importance and control. 1st edition. Pensoft Publishers.

4. Holt RA, Subramanian GM, Halpern A, Sutton GG, Charlab R, et al. (2002) The genome sequence of the malaria mosquito Anopheles gambiae. Science 298: 129-149. PMID: 12364791

5. Nene V, Wortman JR, Lawson D, Haas B, Kodira C, et al. (2007) Genome sequence of Aedes aegypti, a major arbovirus vector. Science 316: 1718-1723. PMID: 17510324 
6. Arensburger P, Megy K, Waterhouse RM, Abrudan J, Amedeo P, et al. (2010) Sequencing of Culex quinquefasciatus establishes a platform for mosquito comparative genomics. Science 330: 86-88. doi: 10.1126/science.1191864 PMID: 20929810

7. Bartholomay LC, Waterhouse RM, Mayhew GF, Campbell CL, Michel K, et al. (2010) Pathogenomics of Culex quinquefasciatus and meta-analysis of infection responses to diverse pathogens. Science 330: 88-90. doi: 10.1126/science.1193162 PMID: 20929811

8. Lewin HA, Larkin DM, Pontius J, O'Brien SJ (2009) Every genome sequence needs a good map. Genome Res 19: 1925-1928. doi: 10.1101/gr.094557.109 PMID: 19596977

9. Dennhofer L (1975) Inherited preferential segregation in translocation heterozygotes of the mosquito, Culex pipiens L. Theor Appl Genet 45: 250-253. doi: 10.1007/BF00831896 PMID: 24419468

10. Mori A, Severson DW, Christensen BM (1999) Comparative linkage maps for the mosquitoes (Culex pipiens and Aedes aegypti) based on common RFLP loci. J Hered 90: 160-164. PMID: 9987925

11. Mori A, Romero-Severson J, Severson DW (2007) Genetic basis for reproductive diapause is correlated with life history traits within the Culex pipiens complex. Insect Mol Biol 16: 515-524. PMID: 17635616

12. Hickner PV, Mori A, Chadee DD, Severson DW (2013) Composite linkage map and enhanced genome map for Culex pipiens complex mosquitoes. J Hered 104: 649-655. doi: 10.1093/jhered/est040 PMID: 23846985

13. Zambetaki A, Pasteur N, Mavragani-Tsipidou P (1998) Cytogenetic analysis of Malpighian tubule polytene chromosomes of Culex pipiens (Diptera: Culicidae). Genome Biol 41: 751-755.

14. Campos J, Andrade CF, Recco-Pimentel SM (2003) Malpighian tubule polytene chromosomes of Culex quinquefasciatus (Diptera, Culicinae). Mem Inst Oswaldo Cruz 98: 383-386. PMID: 12886420

15. McAbee RD, Christiansen JA, Cornel AJ (2007) A detailed larval salivary gland polytene chromosome photomap for Culex quinquefasciatus (Diptera: Culicidae) from Johannesburg, South Africa. J Med Entomol 44: 229-237. PMID: 17427691

16. Dennhofer $L$ (1968) Salivary gland chromosomes of the mosquito Culex pipiens. I. The normal chromosome complement. Chromosoma 25: 365-376. PMID: 5711092

17. Heyse D, Catalan J, Nance E, Britton-Davidian J, Pasteur N (1996) Unconventional organization of amplified esterase $B$ gene in insecticide-resistant mosquitoes of the Culex pipiens complex. J Am Mosq Control Assoc 12: 199-205. PMID: 8827593

18. Sharakhova MV, Timoshevskiy VA, Yang F, Demin S, Severson DW, et al. (2011) Imaginal discs: a new source of chromosomes for genome mapping of the yellow fever mosquito Aedes aegypti. PLoS Negl Trop Dis 5: e1335. doi: 10.1371/journal.pntd.0001335 PMID: 21991400

19. Timoshevskiy VA, Severson DW, Debruyn BS, Black WC, Sharakhov IV, et al. (2013) An integrated linkage, chromosome, and genome map for the yellow fever mosquito Aedes aegypti. PLoS Negl Trop Dis 7: e2052. doi: 10.1371/journal.pntd.0002052 PMID: 23459230

20. Timoshevskiy VA, Kinney NA, Debruyn BS, Mao C, Tu Z, et al. (2014) Genomic composition and evolution of Aedes aegypti chromosomes revealed by the analysis of physically mapped supercontigs. BMC Biol 12: 27. doi: 10.1186/1741-7007-12-27 PMID: 24731704

21. Rai K (1963) A comparative study of mosquito karyotypes. Ann Entomol Soc Amer 56: 160-170.

22. Coluzzi M, Sabatini A (1967) Cytogenetic observations on species A and B of the Anophles gambaie complex. Parassitologia 9: 73-88.

23. Kumar A, Rai KS (1990) Chromosomal localization and copy number of 18S+28S ribosomal RNA genes in evolutionary diverse mosquitoes (Diptera, Culicidae). Hereditas 113: 277-289. PMID: 2093704

24. Hickner PV, Debruyn B, Lovin DD, Mori A, Saski CA, et al. (2011) Enhancing genome investigations in the mosquito Culex quinquefasciatus via BAC library construction and characterization. BMC Res Notes 4: 358. doi: 10.1186/1756-0500-4-358 PMID: 21914202

25. BEI Resources website. Avalible at: www.beiresources.org. Accessed 2013 June 21.

26. Timoshevskiy VA, Sharma A, Sharakhov IV, Sharakhova MV (2012) Fluorescent in situ hybridization on mitotic chromosomes of mosquitoes. J Vis Exp: e4215.

27. Vector Base website. Avalible at: http://www.vectorbase.org. Accessed 2014 October 24.

28. Demin S, Pleskach N, Svetova M, Solovjeva L (2010) High-resolution mapping of interstitial teleomeric repeats in Syrian hamster metaphase chromosomes. Cytogenetic and Genome Research doi: 10. 1159/000321676: 1-5.

29. NIH website. Available at: http://rsb.info.nih.gov/ij/. Accessed 2014 Septemeber 19.

30. Zeiss website. Available at: http://www.zeiss.de. Accessed 2011 April. 
31. Jimenez LV, Kang BK, deBruyn B, Lovin DD, Severson DW (2004) Characterization of an Aedes aegypti bacterial artificial chromosome (BAC) library and chromosomal assignment of BAC clones for physical mapping quantitative trait loci that influence Plasmodium susceptibility. Insect Mol Biol 13: 37-44. PMID: 14728665

32. Levan A, Fredga K, Sandberg AA (1964) Nomenclature for centromeric positions on chromosomes. Hereditas 52: 201-220.

33. Shaffer LG, Slovak ML, Campbell LJ (2009) An international system for human cytogenetic nomenclature. Basel (Switzerland): Karger.

34. Brown SE, Knudson DL (1997) FISH landmarks for Aedes aegypti chromosomes. Insect Mol Biol 6 : 197-202. PMID: 9099584

35. McDonald PT, Rai KS (1970) Correlation of linkage groups with chromosomes in the mosquito, Aedes aegypti. Genetics 66: 475-485. PMID: 5519652

36. Severson DW, Mori A, Zhang Y, Christensen BM (1994) Chromosomal mapping of two loci affecting filarial worm susceptibility in Aedes aegypti. Insect Mol Biol 3: 67-72. PMID: 7987523

37. Severson DW, Thathy V, Mori A, Zhang Y, Christensen BM (1995) Restriction fragment length polymorphism mapping of quantitative trait loci for malaria parasite susceptibility in the mosquito Aedes aegypti. Genetics 139: 1711-1717. PMID: 7789771

38. Zhong D, Menge DM, Temu EA, Chen H, Yan G (2006) Amplified fragment length polymorphism mapping of quantitative trait loci for malaria parasite susceptibility in the yellow fever mosquito Aedes aegypti. Genetics 173: 1337-1345. PMID: 16624910

39. Bosio CF, Fulton RE, Salasek ML, Beaty BJ, Black WCt (2000) Quantitative trait loci that control vector competence for dengue-2 virus in the mosquito Aedes aegypti. Genetics 156: 687-698. PMID: 11014816

40. Gomez-Machorro C, Bennett KE, del Lourdes Munoz M, Black WCt (2004) Quantitative trait loci affecting dengue midgut infection barriers in an advanced intercross line of Aedes aegypti. Insect Mol Biol 13: 637-648. PMID: 15606812

41. George P, Sharakhova MV, Sharakhov IV (2010) High-resolution cytogenetic map for the African malaria vector Anopheles gambiae. Insect Mol Biol 19: 675-682. doi: 10.1111/j.1365-2583.2010.01025.x PMID: 20609021

42. Sharakhova MV, Hammond MP, Lobo NF, Krzywinski J, Unger MF, et al. (2007) Update of the Anopheles gambiae PEST genome assembly. Genome Biol 8: R5. PMID: 17210077

43. Sharakhova MV, Xia A, Tu Z, Shouche YS, Unger MF, et al. (2010) A physical map for an Asian malaria mosquito, Anopheles stephensi. Am J Trop Med Hyg 83: 1023-1027. doi: 10.4269/ajtmh.2010.100366 PMID: 21036831

44. Sharakhov IV, Serazin AC, Grushko OG, Dana A, Lobo N, et al. (2002) Inversions and gene order shuffling in Anopheles gambiae and A. funestus. Science 298: 182-185. PMID: 12364797

45. Campos J, Andrade CF, Recco-Pimentel SM (2003) A technique for preparing polytene chromosomes from Aedes aegypti (Diptera, Culicinae). Mem Inst Oswaldo Cruz 98: 387-390. PMID: 12886421

46. Severson DW, Meece JK, Lovin DD, Saha G, Morlais I (2002) Linkage map organization of expressed sequence tags and sequence tagged sites in the mosquito, Aedes aegypti. Insect Mol Biol 11: 371378. PMID: 12144703

47. Bachtrog D (2013) Y-chromosome evolution: emerging insights into processes of $Y$-chromosome degeneration. Nat Rev Genet 14: 113-124. doi: 10.1038/nrg3366 PMID: 23329112 